\title{
Survey and Analysis of Age and Antiplatelet/Anticoagulation Medication Status of the Desert Regional Medical Center Emergency Department Patients
}

\author{
Chao Li ${ }^{1,2}$, Yasir R. Khan ${ }^{2}$, Javed Siddiqi $2,3,4,5$ \\ 1. Neurosurgery, College of Osteopathic Medicine, Des Moines University, Des Moines, USA 2. Neurosurgery, Desert \\ Regional Medical Center, Palm Springs, USA 3. Neurosurgery, Riverside University Health System Medical Center, \\ Moreno Valley, USA 4. Neurosurgery, Arrowhead Regional Medical Center, Colton, USA 5. Neurosurgery, California \\ University of Science and Medicine, Colton, USA
}

Corresponding author: Chao Li, clark_li@msn.com

\section{Abstract}

\section{Objective}

To delineate whether the geographical region served by the Desert Regional Medical Center (DRMC) emergency department (ED) in Palm Springs, California has a higher percentage of patients taking antiplatelet and/or anticoagulant medications during the winter season due to the seasonal influx of elderly patients.

\section{Method}

We conducted a prospective study in the patients seen in the DRMC ED between November 12, 2019 and November 14, 2019, and those patients were anonymously surveyed regarding their outpatient use of antiplatelet and anticoagulant medications. Verbal consent for participation was obtained. All the registered patients at the DRMC ED during the prescribed period of time were included. While the patients who were sent home by a triage nurse for non-medical issues or minor ailments requiring no treatment from ED providers and patients who refused to participate or were unable to consent were excluded. Age, gender, medication status including whether on any medication, antiplatelet and anticoagulant use, including dosage, and last dose time were recorded.

\section{Results}

Of the 110 consecutive patients arriving to the ED during the study period, 11 patients were unwilling or unable to provide consent for participation. Of the 99 participating patients, 37 (37\%) were over 65 years old. The most common antiplatelet and/or anticoagulant medication taken by the patients was aspirin (24 patients). Aspirin was most commonly taken as once a day, in a total of 11 patients (11\%). Thirty patients

Received 05/18/2021 Review began 05/27/2021 Review ended 06/15/2021 Published 06/15/2021

๑) Copyright 2021 Li et al. This is an open access article distributed under the terms of the Creative Commons Attribution License CC-BY 4.0., which permits unrestricted use, distribution, and reproduction in any medium, provided the original author and source are credited. were taking one or more antiplatelet or anticoagulant medications.

\section{Conclusion}

ED at a hospital in Palm Springs, California has more elderly visits as compared to the national and California state averages. However, antiplatelet usage in elderly patients in our study was lower than the national average.

Categories: Preventive Medicine, Neurosurgery, Public Health

Keywords: antiplatelet, anticoagulation, emergency department, age, aspirin

\section{Introduction}

Palm Springs, California is a metropolitan city with a summertime population of 400,000 . In the winter months, the population has been shown to swell up to 800,000 people, largely due to tourists making shortterm visits and longer-term inhabitants known as seasonal "snowbirds" from northern states and countries [1]. Desert Regional Medical Center (DRMC) is a Joint Commission-certified primary stroke center and is the only Level II trauma center in the Coachella Valley, covering more than 8000 square miles. This dynamic demographic change makes DRMC, the area's only comprehensive stroke center, one of the busiest stroke centers in California, particularly during the winter season. The emergency department (ED) is the main healthcare access point for DRMC's stroke patients, most of whom arrive directly to the ED as a stroke code/alert case or as a transfer from an outside hospital.

For decades, antiplatelet and/or anticoagulation medications, such as aspirin, have been used for treatment and/or prevention of stroke, myocardial infarction, and other cardiac conditions [2]. Almost over 50\% of 


\section{Cureus}

elderly patients have been found taking aspirin daily or every other day with/without the recommendation of a physician. The most recent study shows that over 30 million patients in the USA take aspirin [2]. Notably, antiplatelet and anticoagulant medications increase the risk of cerebral hemorrhage [2]. This paper looks into whether the ED visits in a community hospital differ by age, as well as antiplatelet and anticoagulant use, from the national and state level.

\section{Materials And Methods}

This study was approved by the Institutional Review Board. Patients were given a hard copy with information about the study. All patients who came to the ED were asked to fill out the survey. Patients were excluded if they were sent home by a triage nurse for non-medical issues or minor ailments not requiring the attention of a physician such as prescription refill, headache without warning symptoms, minor injury due to sports, skin abrasion, minor fever, and so on, and patients who refused to participate or were unable to provide consent due to their medical or mental status.

Data collected included age, gender, general medication status, current antiplatelet and anticoagulant medication use, and antiplatelet and anticoagulant dose.

Descriptive statistics were run. Fisher's exact test, chi-square test, or analysis of variance, as applicable, was done to compare groups.

\section{Results}

The study included 99 patients. Table 1 shows the patients' age and gender details.

\begin{tabular}{|l|l|}
\hline Age & Number \\
\hline$<21$ & 12 \\
$21-60$ & 44 \\
$61-80$ & 32 \\
$>80$ & 11 \\
Gender & \\
Male & 62 \\
Female & 37 \\
\hline
\end{tabular}

TABLE 1: Subjects' categorization by age and gender

Table 2 shows the number of subjects who were on general medication, and antiplatelet and anticoagulant medications. 


\section{Cureus}

Medications

Currently not on any medication

Currently on medication but no antiplatelet or anticoagulants

Currently on medication with antiplatelet and/or anticoagulants

Aspirin $81 \mathrm{mg}$ every day

Aspirin $81 \mathrm{mg}+$ clopidogrel $75 \mathrm{mg}$ every day

Rivaroxaban 20 mg every day

Aspirin $81 \mathrm{mg}$ every other day

Aspirin $81 \mathrm{mg}+$ rivaroxabon $20 \mathrm{mg}$

Aspirin 325 mg every day

Aspirin 325 mg + clopidogrel 75 mg every day

Clopidogrel 75 mg twice a day

Aspirin $81 \mathrm{mg}+$ apixaban $5 \mathrm{mg}$ every day

Apixaban $5 \mathrm{mg}$ twice a day

Dabigatran $150 \mathrm{mg}$ twice a day

Aspirin + dabigatran $150 \mathrm{mg}$ twice a day and clopidogrel $75 \mathrm{mg}$ twice a day

TABLE 2: Subjects' medication status as well as dosage of antiplatelet and anticoagulant medications

Aspirin usage was analyzed separately. This included patients who were taking aspirin alone or with other anticoagulants (Table 3).

\begin{tabular}{|l|l|}
\hline Age & Number of subjects taking aspirin (\%) \\
\hline$<18$ & $0(0)$ \\
$18-60$ & $7(7.07)$ \\
$61-80$ & $14(14.14)$ \\
$>80$ & $3(3.3)$ \\
\hline Total & $24(24.24)$ \\
\hline
\end{tabular}

\section{TABLE 3: Aspirin usage by age}

There was a statistical significance found between the age groups $(P=0.006)$, though there was no significance found in the groups when compared to numbers from another study [2].

\section{Discussion}

The study shows that the percentage (37\%) of elderly patients visiting the DRMC ED is higher than the national average for ED visitors in this demographic. A 2012 study by the Centers for Disease Control and Prevention demonstrated that the national average percentage of ED visits made by elderly patients aged 65 years and older was $16 \%$, with the percentage of ED visits made by elderly persons in California at that time being $22 \%$ [3].

Additionally, the number of patients visiting the ED in our survey who were less than 18 years old totaled 9 
(9\%), significantly lower than the national average of $21 \%$ and the California average of $19 \%$ [3]. Despite the higher percentage of elderly patients in our study, the usage of aspirin in this patient group was lower compared to the national average [4].

There was a statistical significance found between the age groups taking aspirin, either alone or in combination with other anticoagulants $(\mathrm{P}<0.05)$. This significance was anticipated since aspirin is primarily used for prevention of stroke and cardiovascular diseases after 40-50 years of age [2,4,5].

There are several limitations of this study. The brevity of the time window during which the survey was performed, and the limited number of patients surveyed may have impacted our results. There are no published data regarding specific anticoagulation usage in general population in state or national level in the USA. The studies we found focus on either specific group such as skilled nursing home residents or comparison between various anticoagulation medication usage in the USA [6,7]. As such, we plan to start a multiple-phase study to follow our initial findings. In phase 2, we will survey another 100 patients over the age of 65 years consecutively arriving to the DRMC ED to assess their medication status, including antiplatelet and anticoagulant medications. In phase 3, 100 non-traumatic intraparenchymal hemorrhage patients consecutively arriving to the DRMC ED will be surveyed regarding their antiplatelet and anticoagulant medication use. The final goal of the study will be to investigate if the rate of antiplatelet and anticoagulant medication use among elderly patients in the California Coachella Valley exceeds that of the national average, and furthermore, if there is a correlation between higher usage of these medications among this population and non-traumatic intraparenchymal hemorrhage.

\section{Conclusions}

DRMC ER patients have a higher percentage of elderly patients compared to the national and California average of patients visiting the ED . However, despite the higher percentage of elderly patients, the prevalence of aspirin usage in our study was lower than the national average.

\section{Additional Information \\ Disclosures}

Human subjects: Consent was obtained or waived by all participants in this study. Metrowest Institutional Review Board issued approval 2019152. This is to inform you that on November 12, 2019 MetroWest Medical Center Institutional Review Board (IRB), via Expedited Review by the IRB Chair, has approved the abovereferenced research protocol and the participation of the above-referenced investigative site in the research. Animal subjects: All authors have confirmed that this study did not involve animal subjects or tissue. Conflicts of interest: In compliance with the ICMJE uniform disclosure form, all authors declare the following: Payment/services info: All authors have declared that no financial support was received from any organization for the submitted work. Financial relationships: All authors have declared that they have no financial relationships at present or within the previous three years with any organizations that might have an interest in the submitted work. Other relationships: All authors have declared that there are no other relationships or activities that could appear to have influenced the submitted work.

\section{References}

1. Palm Springs Economic Report. (2017). Accessed: August 4, 2020: https://www.palmspringsca.gov/home/showdocument.

2. O'Brien CW, Juraschek SP, Wee CC: Prevalence of aspirin use for primary prevention of cardiovascular disease in the United States: results from the 2017 National Health Interview Survey. Ann Intern Med. 2019, 171:596-8. 10.7326/M19-0953

3. Hing E, Rui P: Emergency Department Use in the Country's Five Most Populous States and the Total United States, 2012. National Center for Health Statistics, Hyattsville, MD; 2016.

4. Statistical Brief \# 179: Aspirin Use among the Adult U.S. Noninstitutionalized Population, with and without Indicators of Heart Disease, 2005. (2007). Accessed: August 13, 2020: https://meps.ahrq.gov/data files/publications/st179/stat179.pdf.

5. Stuntz M, Bernstein B: Recent trends in the prevalence of low-dose aspirin use for primary and secondary prevention of cardiovascular disease in the United States, 2012-2015. Prev Med Rep. 2017, 5:183-6. 10.1016/j.pmedr.2016.12.023

6. Kirley K, Qato DM, Kornfield R, Stafford RS, Alexander GC: National trends in oral anticoagulant use in the United States, 2007 to 2011. Circ Cardiovasc Qual Outcomes. 2012, 5:615-21. 10.1161/CIRCOUTCOMES.112.967299

7. Alcusky M, McManus DD, Hume AL, Fisher M, Tjia J, Lapane KL: Changes in anticoagulant utilization among United States nursing home residents with atrial fibrillation from 2011 to 2016. J Am Heart Assoc. 2019, 8:e012023. 10.1161/JAHA.119.012023 